\title{
Quantifying the Atomic Ordering of Binary Intermetallic Nanocatalysts Using In Situ Heating STEM and XRD
}

\author{
Yao Yang, ${ }^{1}$ Yin Xiong, ${ }^{1}$ Elliot Padgett, ${ }^{2}$ Howie Joress, ${ }^{3}$ Venkata Yarlagadda, ${ }^{4}$ Anusorn Kongkanand, ${ }^{4}$ \\ Joel D. Brock, ${ }^{3}$ Francis J. DiSalvo, ${ }^{1}$ Héctor D. Abruña, ${ }^{1}$ David. A. Muller. ${ }^{2, *}$ \\ 1. Department of Chemistry and Chemical Biology, Cornell University, Ithaca, NY 14853, USA. \\ 2. School of Applied and Engineering Physics, Cornell University, Ithaca, NY 14853, USA. \\ 3. Cornell High Energy Synchrotron Source, Cornell University, Ithaca, NY 14853, USA. \\ 4. Fuel Cell R\&D, General Motors Global Propulsion Systems, Pontiac, MI 48340, USA. \\ *Corresponding author: dm24@cornell.edu
}

Platinum-based intermetallics display superior electrocatalytic activity for the normally sluggish oxygen reduction reaction (ORR), enabling a decrease in $\mathrm{Pt}$ loading compared to $\mathrm{Pt} / \mathrm{C}$ in proton exchange membrane fuel cells (PEMFCs) [1-2]. As-synthesized, Pt-based metal alloy (Pt-M) nanoparticles (NPs) generally form as disordered alloys. The disordered alloys often suffer from a rapid loss of activity during electrochemical cycling due to the dissolution of the $\mathrm{M}$ element and subsequent changes in both the particle morphology and crystal structure. After post-synthesis annealing at high temperatures, the disordered alloy can be transformed into the ordered intermetallic phase, which can show significantly enhanced durability and activity. However, annealing can increase the particle size, which is undesirable. To optimize performance, we must find a balance between increased ordering and particle size.

Here we report quantitative in-situ annealing studies [3], correlating the degree of ordering in intermetallics using XRD with the evolution of particle morphology and the formation of ordered intermetallics, at the atomic scale, using STEM. In situ heating, synchrotron-based XRD was performed to investigate the impact of a variety of annealing conditions on the degree of ordering of large ensembles of $\mathrm{Pt}_{3} \mathrm{Co}$ NPs (Figure 1A). In general, a higher degree of ordering leads to more durable electrocatalysts (Figure 1B). In situ heating STEM was employed to directly visualize the morphological changes (Figures 2A-F), and measure the size distribution of thousands of particles (Figures 2A-H), using an automated algorithm [4]. The relative $\mathrm{Pt}_{3} \mathrm{Co}$ specific surface area (SSA) loss was calculated from STEM images to be around 33\%, which is consistent with the loss in electrochemical surface area (ECSA) measured by cyclic voltammetry (CV) (Figures 2I). STEM images confirm the formation of both fully and partially ordered NPs at the atomic scale (Figures $2 \mathrm{~J}-\mathrm{K}$ ). The annealed $\mathrm{Pt}_{3} \mathrm{Co} / \mathrm{C}$, with an optimal degree of ordering identified at $750{ }^{\circ} \mathrm{C}$, exhibited significantly enhanced durability in practical membrane electrode assembly (MEA) measurements. The results highlight the importance of understanding the annealing process to optimize electrocatalytic activity [5].

References:

[1] D. Wang, H. Xin, et. al., D. A. Muller, F. J. DiSalvo, H. D. Abruña, Nat. Mater. 12 (2013), p. 81-87.

[2] Y. Xiong, Y. Yang, F. J. DiSalvo, H. D. Abruña, J. Am. Chem. Soc. 140 (2018), p. 7248-7255.

[3] Y. Xiong, Y. Yang, H. Joress, E. Padgett, e t. al., J. D. Brock, F. J. DiSalvo, D. A. Muller, H. D. Abruña, Proc. Natl. Acad. Sci. U.S.A. 116 (2019), p. 1974-1983.

[4] E. Padgett, et al., D. A. Muller. J. Electrochem. Soc. 166 (2019), p. F198-F207. 
[5] Work funded by U.S. DOE grant DE-EE0007271. TEM facility supported by NSF MRSEC program (DMR-1719875) and NSF MRI grant (DMR-1429155). XRD supported by NSF Award DMR-1332208.
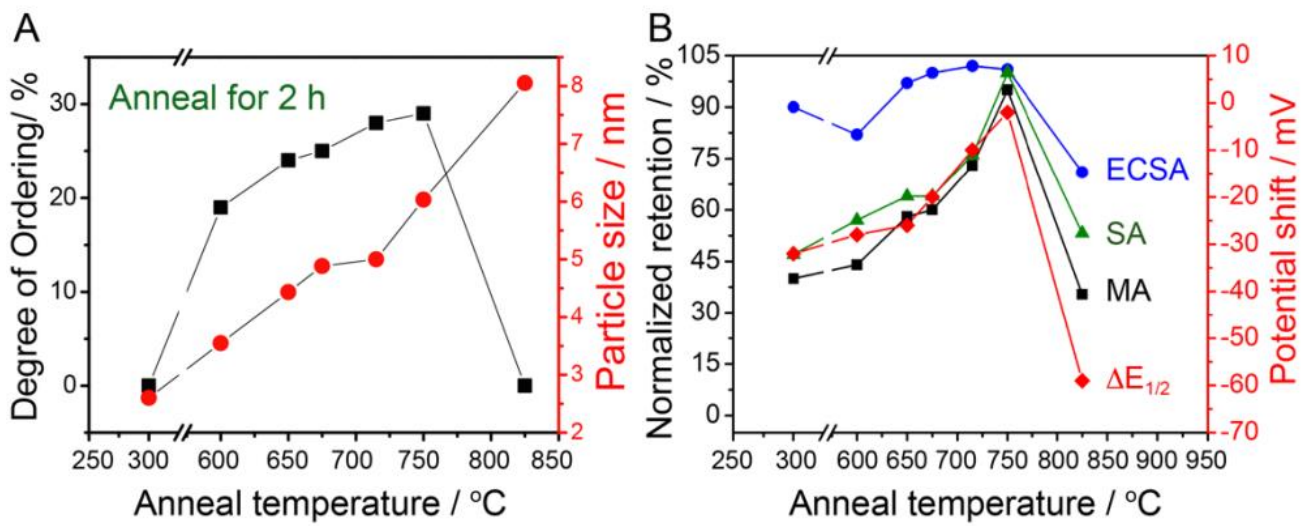

Figure 1. (A) Degree of ordering and crystallite size of $\mathrm{Pt}_{3} \mathrm{Co} / \mathrm{C}$ at various annealing temperatures, measured from in situ heating XRD patterns, showing an optimal degree of ordering at $750{ }^{\circ} \mathrm{C}$. (B) Normalized catalyst activity and ECSA retention as a function of annealing temperature. Mass activity (MA), surface activity (SA) and ECSA retention was calculated based on the ratio of the initial activity/surface areas to that after durability tests. The best durability was achieved at $750{ }^{\circ} \mathrm{C}$, corresponding to the optimal degree of ordering.
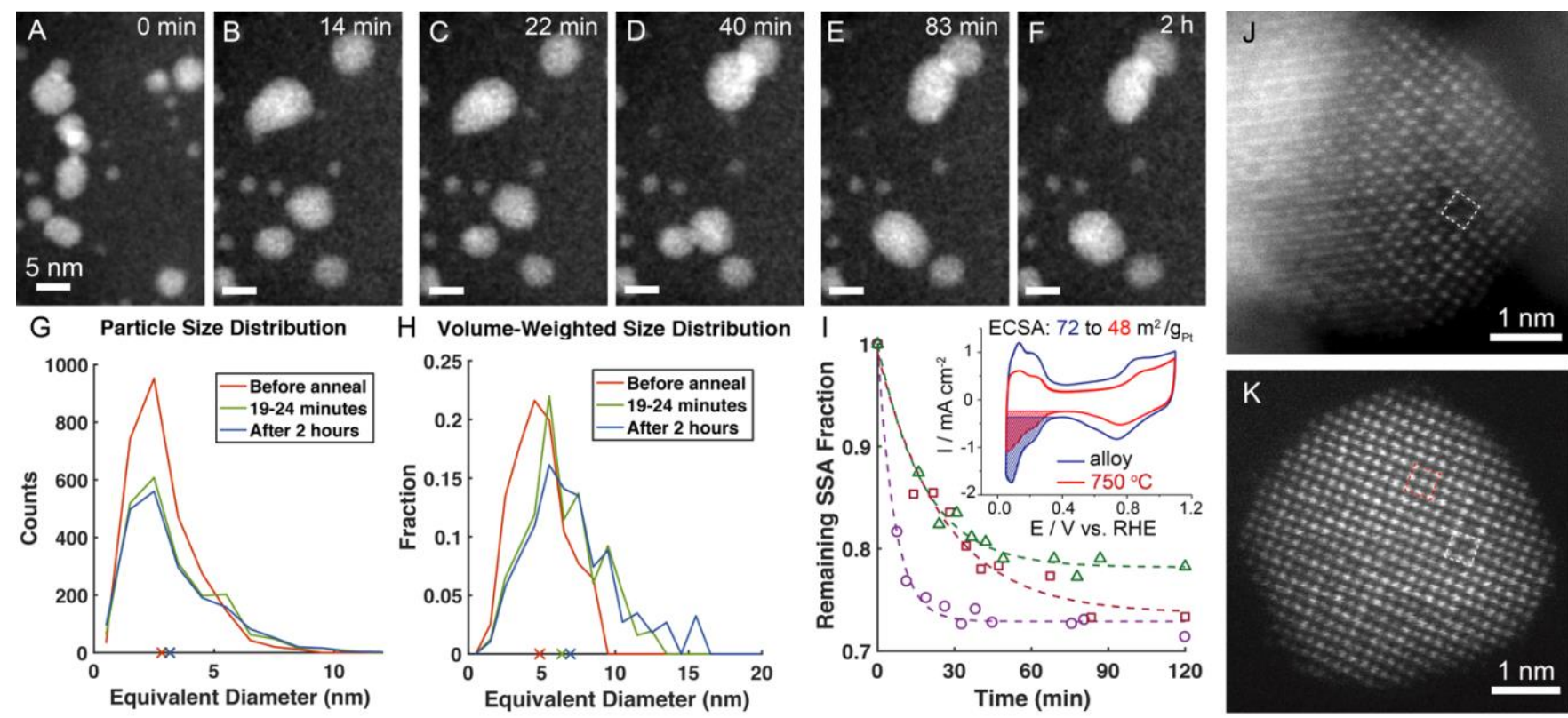

Figure 2. (A-F) In situ heating STEM tracking of the morphological changes of $\mathrm{Pt}_{3} \mathrm{Co} / \mathrm{C}$ at $750{ }^{\circ} \mathrm{C}$, showing the particle migration and coalescence during a $2 \mathrm{~h}$ annealing process. Scale bars: A-F, $5 \mathrm{~nm}$. (G) Measured particle size distribution of thousands of particles in STEM images [3]. (H) Volume-weighted particle size distribution, reflecting the relative contribution of larger particles more clearly. (I) Relative SSA loss of $\mathrm{Pt}_{3} \mathrm{Co}$ estimated from STEM images of three different regions during annealing. Inset shows the ECSA of $\mathrm{Pt}{ }_{3} \mathrm{Co} / \mathrm{C}$ before and after annealing, measured from $\mathrm{CV}$ profiles. (J-K) Atomic-scale ex-situ STEM images of $\mathrm{Pt}_{3} \mathrm{Co} / \mathrm{C}$ after annealing. (J) An ordered intermetallic $\mathrm{Pt}_{3} \mathrm{Co}$ particle on the [100] zone axis. Brighter atoms at corners of squares are $\mathrm{Pt}$, and dimmer atoms at the center are Co (white boxes). (K) A partially ordered intermetallic particle on the [100] zone axis. Both partially-ordered (red boxes) and ordered (white boxes) regions are noted. 